\title{
The Unified Description of a Solution-Particle
}

\author{
Tafireyi Nemaura1,2 \\ ${ }^{1}$ Department of Applied Mathematics, National University of Science and Technology, Bulawayo, Zimbabwe \\ ${ }^{2}$ Department of Clinical Pharmacology, University of Zimbabwe, Harare, Zimbabwe \\ Email: tnemaura@gmail.com, tafireyi.nemaura@nust.ac.zw
}

How to cite this paper: Nemaura, T. (2017) The Unified Description of a Solution-Particle. Journal of Applied Mathematics and Physics, 5, 991-1000. https://doi.org/10.4236/jamp.2017.55086

Received: March 30, 2017

Accepted: May 9, 2017

Published: May 12, 2017

Copyright $\odot 2017$ by author and Scientific Research Publishing Inc. This work is licensed under the Creative Commons Attribution International License (CC BY 4.0).

http://creativecommons.org/licenses/by/4.0/

\begin{abstract}
This work derives a unified description of a solution-particle using movement rate of component vibrations (strings). Projected Pharmacokinetic and Pharmacodynamic data from a patient on Efavirenz are used. Differential equations are used to formulate a system that governs a solution-particle field. A reconnaissance (frame) wave that envelops a solution-particle is derived. Its conductivity and diffusivity fluxes are found. The movement of the reconnaissance wave is found to be faster than diffusion and pilot waves. A solution-particle is accompanied by the three waves, diffusion, pilot and the frame and is active only at the boundary of space and time.
\end{abstract}

\section{Keywords}

Deterministic Theory, Unification, Space and Time Boundary, Time

\section{Introduction}

The unified description of a particle has interested physicists and mathematicians for over a century. This has given rise to different theories in quantum mechanics [1]. Einstein understood the need for a deterministic framework of a quantum mechanics theory and attempted unification of the field [1] [2] [3]. Bohm realised the shortcomings of basing the behaviour of a particle to be wholly explained by the wave, which is underpinned by probability and gave a possible interpretation [4]. In this work, we give a new deterministic approach to the problem. There is a suggestion of the main entities that comprise the "particle" by considering a solution-particle in time [6] [7] [8]. There is consideration of solution-particle formation in time in blood-plasma. This projection is done in an individual who had been on a drug efavirenz [6] [7] [8].

Time is an existence continuous virtual fabric that transmits energy/matter and apparent flux-entities in this dimension form three unstable systems and one outcrop stable system parallel to space. Time follows a one to one relationship with the space and time boundary that is the map between time and space 
and time boundary is morphism. A system is unique in time and consequently at the boundary of space and time (space-time) this is primarily because of the uniqueness of time. Furthermore, we obtain an "apparent enveloping" unstable field (frame wave) that interacts with the boundary of space and time. Time can be described as the complimentary-concentration of virtual matter. A solutionparticle is only active on the space and time boundary. A solution-particle is a product in space and time and resides at the boundary of space and time. Space is real while time is virtual.

Diffusion (gradient driven) and other wave processes are unstable virtual particles that propagate matter-in-time. While concentration is an anti-solvation movement propagation measure of matter and apparent flux-entities in this dimension form a stable system. The time system consists of diffusion (inter), derived solution-particle (in), pilot wave (extra) and reconnaissance (frame) movement systems associated with a solution particle on the boundary of space and time. The solution particle consists of an intra-movement system. A solution-particle is apparently governed by a $3+1$ parts independent system, a stable single concentration-component $(x(t))$ (which has a derived movement state in time) and the accompanying three unstable time-component parts that are the one that aides diffusion and the other wave forms. The time-component establishes/maintains equilibrium of the concentration-component with the immediate environment of a solution-particle at space-time.

We describe the three part-system as comprising of diffusion an abscissa unstable four dimensional radial movement driven by gradient, pilot wave an ordinate unstable four dimensional radial movement driven by tension and solution-particle an applicate stable three dimensional radial movement, in addition to the frame wave form. Diffusion and the other waves are tracked in the time plane. These are particles of matter-in-time. The pilot wave movement is vertical in time and acts on the non-dissipative part of time. Furthermore, diffusion is horizontal with respect to dissipative part of time. The solution-particle is defined in time.

In this work, we attempt to establish the continuity of a solution-particle in time, and introducing the frame wave that governs the solution particle and the other two waves (Diffusion and Pilot). There is an investigation and a proposal of the form of the waves associated with an active solution-particle in time and their cooperative relationship including the stability of the inter-system of waves and the solution-particle.

\section{Methods}

Projected PK/PD data of a patient on $60 \mathrm{mg}$ of efavirenz is used [6] [7]. Ordinary differential equations and nonlinear regression models are used. The software used, were R and Mathematica.

\subsection{Description of the Apparent System in Time}

A model is proposed that does extend relations between apparent entities in the 
apparent system. There is consideration of three important independent phases that sorrounds a solution-particle in time. The three orthogonal part-system, $\Delta_{0}$-derived intra-movement system in a solution particle (Secondary System), $\Lambda_{1}$-inter-movement system of a solution particle driven by gradient in solution particles [7], and $\Lambda_{1^{\prime}}$-extra-movement system of a solution particle driven by tension in solution particles [6]. The three orthogonal part systems are such that,

$$
\Lambda_{1}(t)+\Delta_{0}(t)+\Lambda_{1^{\prime}}(t)=0,
$$

with

$$
\Lambda_{1}(t)=\Delta_{0}(t)=\Lambda_{1^{\prime}}(t)=0, \forall t,
$$

where

$$
\begin{gathered}
\Lambda_{1}(t)=A_{1}(t)+S_{1}(t)+\bar{P}_{1}(t)+\bar{C}_{1}(t), \\
\Delta_{0}(t)=\bar{C}_{0}(t)+A_{0}(t)+S_{0}(t), \quad \bar{P}_{0}(t):=0,
\end{gathered}
$$

and

$$
\Lambda_{1^{\prime}}(t)=A_{1^{\prime}}(t)+S_{1^{\prime}}(t)+\bar{P}_{1^{\prime}}(t)+\bar{C}_{1^{\prime}}(t) .
$$

The functions $C_{i}, S_{i}, P_{i}$ and $A_{i}$ are projected to have the form

$$
\begin{gathered}
C_{i}(t)=\alpha_{c_{i}} \mathrm{e}^{-\beta_{c_{i}} t}, \\
S_{i}(t)=\frac{\alpha_{s_{i}} t}{\beta_{s_{i}}+t}, \\
P_{i}(t)=\alpha_{p_{i}}\left(1-\mathrm{e}^{-\beta_{p_{i}} t}\right),
\end{gathered}
$$

and

$$
A_{i}(t)=C_{i}(t)+\bar{S}_{i}(t)+P_{i}(t),
$$

where $i=1,0,1^{\prime}$ and $A_{i}, S_{i}, P_{i}$ and $C_{i}$ are Advective, Saturation, Passive and Convective components respectively associated with the $\mathrm{i}^{\text {th }}$ movement system. Furthermore $\alpha_{c_{i}}, \beta_{c_{i}}, \alpha_{s_{i}}, \beta_{s_{i}}, \alpha_{p_{i}}$ and $\beta_{p_{i}}$ are constants. We consider the derived secondary saturation movement [7], which estimates the total anti-solvation $\left(A_{0}+S_{0}\right)$ (equivalent to the pro-solvation movement $C_{0}(t)$ ),

$$
F(t)=v_{r}\left(\mathrm{e}^{-v_{b} t}-\mathrm{e}^{-v_{a} t}\right) \approx C_{0}(t), t \in[0,24]
$$

where, $t$ is time and $v_{a}, v_{b}$ and $v_{r}$ are constants. The time $(t)$ variable does not necessarily stop at 24 hours. This is only considered because it is the dosing interval of the drug efavirenz. With $F(t) \approx A_{0}(t)+S_{0}(t)=C_{0}(t)$. The state variable $F(t)$ is the derived total anti-solvation movement in time of concentration in time. It is obtained by considering concentration in time $(x(t))$ as tracked by the secondary saturation measure [7].

\subsection{The Apparent Enveloping Form System in Time $\left(\Omega_{\infty}\right)$}

We make use of the total anti-solvation component $A_{0}+S_{0}\left(=C_{0}\right)$ of the frame system associated with solution-particle. In addition, we formulate a model in time for the relationship between diffussion and pilot wave with the total antisolvation derived movement of the solution-particle (Figure 1). 


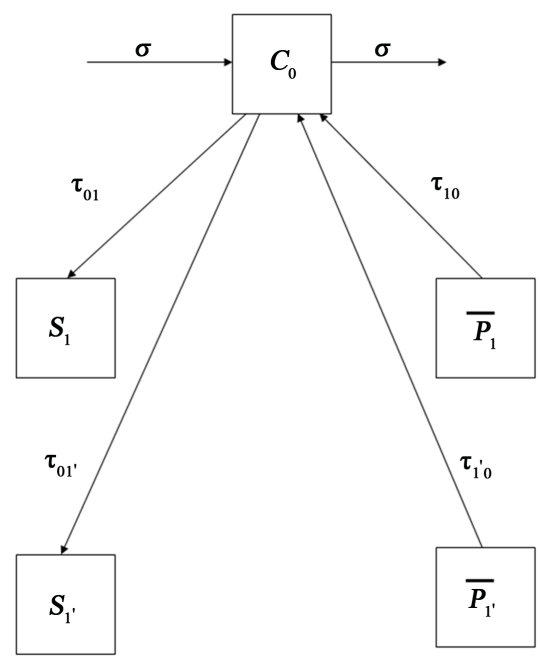

Figure 1. A compartmental representation of the model for movement state dynamics in extracting the frame and the constiuent sub-particles advective forms.

The Saturation and Passive transport state variables of diffusion and pilot wave are used in modelling the frame. This is because of the one to one relation they share with the frame. We construct the inter-system associated with the frame,

$$
\begin{aligned}
\frac{\mathrm{d} C_{0}(t)}{\mathrm{d} t}=\sigma(t)-\sigma(t) C_{0}-\tau_{01}(t) C_{0}+\tau_{10}(t) \bar{P}_{1}-\tau_{01^{\prime}}(t) C_{0}+\tau_{1^{\prime} 0}(t) \bar{P}_{1^{\prime}}, \\
\frac{\mathrm{d} S_{1}}{\mathrm{~d} t}=\tau_{01}(t) C_{0} \\
\frac{\mathrm{d} \bar{P}_{1}}{\mathrm{~d} t}=-\tau_{10}(t) \bar{P}_{1} \\
\frac{\mathrm{d} S_{1^{\prime}}}{\mathrm{d} t}=\tau_{01^{\prime}}(t) C_{0} \\
\frac{\mathrm{d} \bar{P}_{1^{\prime}}}{\mathrm{d} t}=-\tau_{1^{\prime} 0}(t) \bar{P}_{1^{\prime}} .
\end{aligned}
$$

Solving the field system (11)-(15) in terms of advective components,

$$
\sigma(t)=\frac{\frac{\mathrm{d} C_{0}}{\mathrm{~d} t}+\tau_{01}(t) C_{0}-\tau_{10}(t) \bar{P}_{1}+\tau_{01^{\prime}}(t) C_{0}-\tau_{1^{\prime} 0}(t) \bar{P}_{1^{\prime}}}{1-C_{0}},
$$

where,

$$
\begin{gathered}
\tau_{01}(t)=\frac{\frac{\mathrm{d} S_{1}}{\mathrm{~d} t}}{C_{0}}, \\
\tau_{10}(t)=\frac{\frac{\mathrm{d} \bar{P}_{1}}{\mathrm{~d} t}}{-\overline{P_{1}}}, \\
\tau_{01^{\prime}}(t)=\frac{\frac{\mathrm{d} S_{1^{\prime}}}{\mathrm{d} t}}{C_{0}},
\end{gathered}
$$




$$
\tau_{1^{\prime} 0}(t)=\frac{\frac{\mathrm{d} \overline{1^{\prime}}}{\mathrm{d} t}}{-\overline{P_{1^{\prime}}}} .
$$

A new system which form part of the frame wave $\left(\Omega_{\infty}\right)$ is given and is such that,

$$
\sigma=\sigma_{\infty}(t)=A_{\infty}(t)=C_{\infty}(t)+P_{\infty}(t)+\bar{S}_{\infty}(t)
$$

thus,

$$
\Omega_{\infty}(t)=A_{\infty}(t)+S_{\infty}(t)+\bar{C}_{\infty}(t)+\bar{P}_{\infty}(t)=0 .
$$

We present the conceptual framework of a solution-particle-in-time (Figure 2). This shows the three accompanying waves and the solution-particle.

\subsection{Local Analysis of the Inter-Particle Field System with the}

Frame near Steady-State Points $\left(C_{0}^{*}, S_{1}^{*}, \bar{P}_{1}^{*}, S_{1^{\prime}}^{*}, \bar{P}_{1^{\prime}}^{*}\right)$

Setting

$$
\frac{\mathrm{d} C_{0}(t)}{\mathrm{d} t}=\frac{\mathrm{d} S_{1}}{\mathrm{~d} t}=\frac{\mathrm{d} \bar{P}_{1}}{\mathrm{~d} t}=\frac{\mathrm{d} S_{1^{\prime}}}{\mathrm{d} t}=\frac{\mathrm{d} \bar{P}_{1^{\prime}}}{\mathrm{d} t}=0,
$$

we obtain the equilibrium points,

$$
C_{0}^{*}=1, S_{1}^{*}=\bar{P}_{1}^{*}=S_{1^{\prime}}^{*}=\bar{P}_{1^{\prime}}^{*}=0 .
$$

The Jacobian matrix for the field inter-system is given by,

$$
J\left(C_{0}^{*}, S_{1}^{*}, \bar{P}_{1}^{*}, S_{1^{\prime}}^{*}, \bar{P}_{1^{\prime}}^{*}\right)=\left[\begin{array}{ccccc}
-\left(\sigma+\tau_{01}+\tau_{01^{\prime}}\right) & 0 & \tau_{10} & 0 & \tau_{1^{\prime} 0} \\
\tau_{01} & 0 & 0 & 0 & 0 \\
0 & 0 & -\tau_{10} & 0 & 0 \\
\tau_{01^{\prime}} & 0 & 0 & 0 & 0 \\
0 & 0 & 0 & 0 & -\tau_{1^{\prime} 0}
\end{array}\right]_{\left(C_{0}^{*}, S_{1}^{*}, \bar{P}_{1}^{*}, S_{1}^{*}, P_{1^{\prime}}^{*}\right)}
$$

The characteristic equation of the field system is given by,

$$
\lambda^{2}\left(\lambda+\tau_{10}\right)\left(\lambda+\tau_{1^{\prime} 0}\right)\left(\lambda+\sigma+\tau_{01}+\tau_{01^{\prime}}\right)=0,
$$

thus the eigenvalues are $\lambda_{1}=\lambda_{2}=0, \lambda_{3}=-\tau_{10}, \lambda_{4}=-\tau_{1^{\prime} 0}, \lambda_{5}=-\left(\sigma+\tau_{01}+\tau_{01^{\prime}}\right)$.

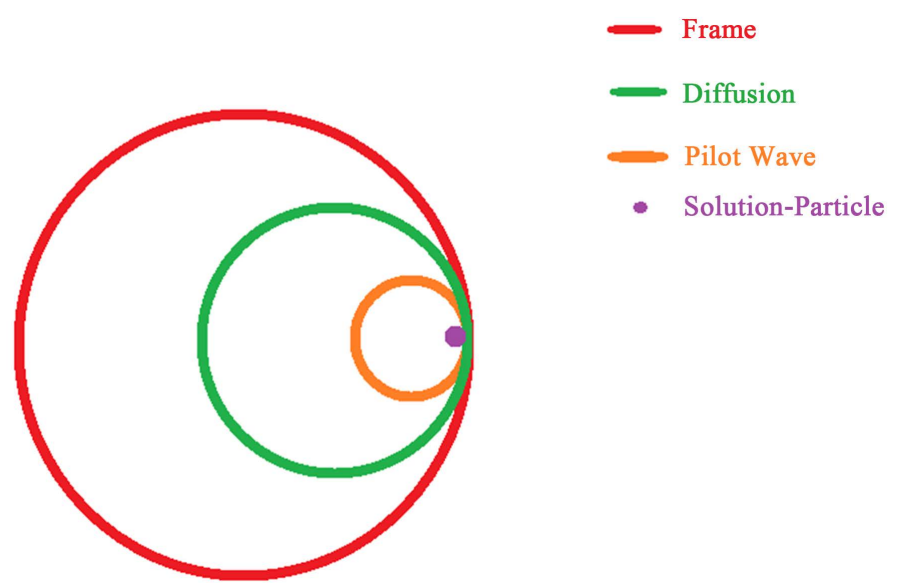

Figure 2. A unified conceptual representation of the solution-particle in time. 


\section{Results}

\subsection{Numerical Movement Rates of the Inter-System to the Frame}

We take into consideration the state variables involved in modelling the Frame. In addition, to the estimation of parameters of $A_{0}+S_{0}\left(=C_{0}\right)$, there are also parameter estimates for state variables $S_{1}$ and $\bar{P}_{1}$ (components of diffusion) and $S_{1^{\prime}}$ and $\bar{P}_{1^{\prime}}$ (components of pilot wave) (Table 1).

There is consideration of the frame's inter-system with the other systems and its constituent sub-particles. The sub-particles $(\tau)$ movement system represented by Equations (17)-(20) holding the field's inter-system are obtained and the estimated fitted equations are given by,

$$
\begin{aligned}
& \tau_{01}(t)=\frac{\theta_{1}^{(01)}}{t^{(01)}}+\theta_{1}^{(01)} \mathrm{e}^{-\theta_{3}^{(01)} t}, \\
& \tau_{10}(t)=\frac{\theta_{1}^{(10)}}{t^{\theta_{2}^{(10)}}}+\theta_{1}^{(10)} \mathrm{e}^{-\theta_{3}^{(10)} t}, \\
& \tau_{01^{\prime}}(t)=\frac{\theta_{1}^{\left(01^{\prime}\right)}}{t^{\theta_{2}^{\left(01^{\prime}\right)}}}+\theta_{1}^{\left(01^{\prime}\right)} \mathrm{e}^{-\theta_{3}^{\left(01^{\prime}\right)}}, \\
& \tau_{1^{\prime} 0}(t)=\frac{\theta_{1}^{\left(1^{\prime} 0\right)}}{t^{\theta_{2}^{\left(1^{\prime} 0\right)}}}+\theta_{1}^{\left(1^{\prime} 0\right)} \mathrm{e}^{-\theta_{3}^{\left(1^{\prime} 0\right)} t} .
\end{aligned}
$$

The estimated parameters of Equations (26)-(29) are given (Table 2).

\subsection{Frame Conductivity and Diffusivity Flux}

Using solutions to Equations (17)-(20) and substituting into Equation (16)

\begin{tabular}{|c|c|c|c|c|c|}
\hline Advective & Parameters & Estimate & Std Error & $t$ value & $\operatorname{Pr}(>|t|)$ \\
\hline \multirow{3}{*}{$F(t) \approx C_{0}(t)$} & $v_{r}$ & 0.623106 & 0.012214 & 51.02 & $<2 \times 10^{-16}$ \\
\hline & $v_{b}$ & 0.022465 & 0.001203 & 18.68 & $5.35 \times 10^{-15}$ \\
\hline & $v_{a}$ & 0.439258 & 0.017354 & 25.31 & $<2 \times 10^{-16}$ \\
\hline \multirow{2}{*}{$S_{1}(t)$} & $\alpha_{s_{1}}$ & 99.406672 & 13.520844 & 7.352 & $5.74 \times 10^{-7}$ \\
\hline & $\beta_{s_{1}}$ & 1.979299 & 0.179995 & 10.996 & $1.12 \times 10^{-9}$ \\
\hline \multirow{2}{*}{$\bar{P}_{1}(t)$} & $\alpha_{p_{1}}$ & -87.672322 & 12.141991 & -7.221 & $7.42 \times 10^{-7}$ \\
\hline & $\beta_{p_{1}}$ & 0.157279 & 0.002074 & 75.816 & $<2 \times 10^{-16}$ \\
\hline \multirow{2}{*}{$S_{1^{\prime}}(t)$} & $\alpha_{s_{p_{1}}}$ & 23.527008 & 3.210289 & 7.239 & $6.01 \times 10^{-7}$ \\
\hline & $\beta_{1^{\prime}}$ & 1.632012 & 0.154663 & 10.552 & $2.20 \times 10^{-9}$ \\
\hline \multirow{2}{*}{$\bar{P}_{1^{\prime}}(t)$} & $\alpha_{1^{\prime}}$ & -19.868405 & 2.919178 & -6.806 & $1.69 \times 10^{-6}$ \\
\hline & $\beta_{1^{\prime}}$ & 0.180557 & 0.003487 & 51.783 & $<2 \times 10^{-16}$ \\
\hline
\end{tabular}
through the use of state variables with estimated parameters (Table 3), we obtain the advective component $\sigma(t)$. The following relation is fitted,

Table 1. Parameter estimates of state variables movement rates in modelling the frame. 
Table 2. Parameter estimates in modelling sub-particles movement rates associated with the frame.

\begin{tabular}{|c|c|c|c|c|c|}
\hline Advective & Parameters & Estimate & Std Error & t value & $\operatorname{Pr}(>|\mathbf{t}|)$ \\
\hline & $\theta_{1}^{(01)}$ & 64.0248 & 0.9691 & 66.064 & $<2 \times 10^{-16}$ \\
\hline \multirow[t]{3}{*}{$\tau_{01}$} & $\theta_{2}^{(01)}$ & 3.0711 & 0.3424 & 8.968 & $8.42 \times 10^{-9}$ \\
\hline & $\theta_{3}^{(01)}$ & 0.4078 & 0.0203 & 20.090 & $1.21 \times 10^{-15}$ \\
\hline & $\theta_{1}^{(10)}$ & -0.527814 & 0.004731 & -111.56 & $<2 \times 10^{-16}$ \\
\hline \multirow[t]{3}{*}{$\tau_{10}$} & $\theta_{2}^{(10)}$ & 2.11093 & 0.118748 & 17.78 & $1.54 \times 10^{-14}$ \\
\hline & $\theta_{3}^{(10)}$ & 0.292089 & 0.009347 & 31.25 & $<2 \times 10^{-16}$ \\
\hline & $\theta_{1}^{\left(01^{\prime}\right)}$ & 16.25989 & 0.22766 & 71.422 & $<2 \times 10^{-16}$ \\
\hline \multirow[t]{3}{*}{$\tau_{01^{\prime}}$} & $\theta_{2}^{\left(01^{\prime}\right)}$ & 3.18268 & 0.33554 & 9.485 & $3.13 \times 10^{-9}$ \\
\hline & $\theta_{3}^{\left(01^{\prime}\right)}$ & 0.44634 & 0.02136 & 20.897 & $5.32 \times 10^{-16}$ \\
\hline & $\theta_{1}^{\left(1^{\prime} 0\right)}$ & -0.526384 & 0.00399 & -131.92 & $<2 \times 10^{-16}$ \\
\hline \multirow[t]{2}{*}{$\tau_{1^{\prime} 0}$} & $\theta_{2}^{\left(1^{\prime} 0\right)}$ & 2.048312 & 0.099094 & 20.67 & $6.68 \times 10^{-16}$ \\
\hline & $\theta_{3}^{\left(1^{\prime} 0\right)}$ & 0.313429 & 0.009489 & 33.03 & $<2 \times 10^{-16}$ \\
\hline
\end{tabular}

Table 3. Parameter estimates in modelling frame movement conductivity fluxes in Equation (30).

\begin{tabular}{ccccc}
\hline Parameters & Estimate & Std Error & $t$ value & $\operatorname{Pr}(>|t|)$ \\
\hline$\alpha_{c_{\infty}}$ & 9.137 & 0.3579 & 25.529 & $1.37 \times 10^{-15}$ \\
$\beta_{c_{\infty}}$ & 0.116 & 0.008653 & 13.408 & $8.29 \times 10^{-11}$ \\
$\alpha_{p_{\infty}}$ & -179.1 & 10.28 & -17.424 & $1.03 \times 10^{-12}$ \\
$\gamma_{p_{\infty}}$ & 0.7677 & 0.01489 & 51.572 & $<2 \times 10^{-16}$ \\
$\beta_{p_{\infty}}$ & 0.0625 & 0.008216 & 7.607 & $4.99 \times 10^{-7}$ \\
$\alpha_{s_{\infty}}$ & -185.3 & 13.9 & -13.329 & $9.14 \times 10^{-11}$ \\
$\beta_{s_{\infty}}$ & 3.46 & 0.1762 & 19.634 & $1.32 \times 10^{-13}$ \\
\hline
\end{tabular}

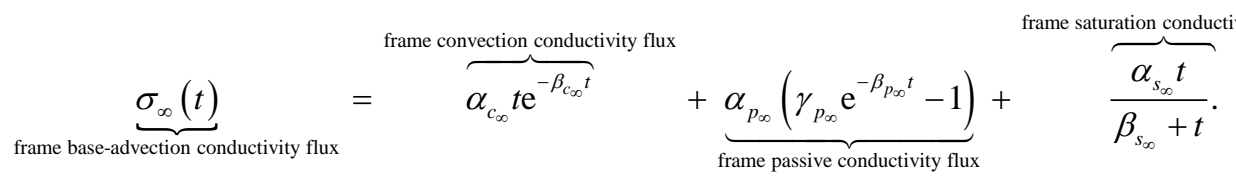

The negative of conductivity flux give rise to diffusivity flux of the frame and is given by

$$
\underbrace{-\sigma_{\infty}(t)}_{\text {frame base-advection diffusivity flux }}=\overbrace{-\alpha_{c_{\infty}} t \mathrm{e}^{-\beta_{c_{\infty}} t}}^{\text {frame convection diffusivity flux }}+\underbrace{\alpha_{p_{\infty}}\left(1-\gamma_{p_{\infty}} \mathrm{e}^{-\beta_{p_{\infty}} t}\right)}_{\text {frame passive diffusivity flux }}+\overbrace{\frac{-\alpha_{s_{\infty}} t}{\beta_{s_{\infty}}+t}}^{\text {frame saturation diffusivity }} \text {. }
$$

It is important to note that in this case for Saturation, 


$$
\left|S_{\infty}(t)\right| \geq\left|S_{1}(t)\right| \geq\left|S_{1^{\prime}}(t)\right| \quad \forall t,
$$

however one can also use Passive movement. The frame wave moves faster than diffusion and the pilot respectively. Considering the stability of the frame's intersystem with the following eigenvalues, $\lambda_{1}=\lambda_{2}=0, \lambda_{3}, \lambda_{4} \geq 0$ and $\lambda_{5} \leq 0$ a.e. $\forall t$. The eigenvalues show that the system is unstable.

\section{Discussion}

Considering a new projection of space-time into the time domain enabled the derivation of waves that accompany a solution particle in time. There is existence of the frame wave that holds the solution particle together. A wave is a four dimensional particle of time consequently space-time and is unstable. However, a solution-particle is stable. The four dimensional systems are unstable while the three dimensional systems are stable [10]. This work shows that the three dimensional solution-particle cannot be considered as a single entity but consists of three important waves through its derived potential state. We construct a potentially unified description of a particle in time. It is shown that the frame's hold on the inter-particle system is unstable.

Time has been redefined in this work to better understand what is possibly occurring. Concisely, time is a unique implicated continuous virtual fabric that frames systems such as matter/energy. At an observation, there is interaction with time (thus waves) and thus nullification of the wave aspects of the particle. The interpretation of quantum mechanics is rooted in the concept of time [9], further to that of the Schrödinger equation. Time is a local parameter with respect to the solution particle however it is non-local with respect to the waves. Waves at their core are abstractions of unstable aspects of time. The concept of space-time gives an interpretation of space as a variable that could be aligned with time. Time is a particle of the virtual fabric and also has occupation potential. The solution-particle-in-time is unique in time thus space-time.

This work agrees with the idea of de-Broglie-Bohm's interpretation of the existence of a quantum potential [4] [11] [12]. In our case, it is the frame and is a wave (de Broglie's Pilot wave). The pilot wave in this work is the one that propels the particle vertically in time. This work has proposed what constitute a wave mathematically. There have been several interpretations of quantum mechanics and several theories which include Copanhagen, Bohm and the Multiple Universe [13]. The movement systems in a solution-particle are vibrations.

This work highlights that the Schrödinger's equation is not sufficient to fully explain what is happening to a particle. There is also diffusion potential of a particle. We returned to formulation of the two equations the diffusion and wave equation and concepts in differential equations to comprehend the possible particles that accompany the solution particle.

When one observes a solution particle it is the only particle defined and all the other particles are unstable in time (waves). This includes the three-wave diffusion, pilot and the frame and they are four dimensional movement components. The issue of differences of kinetic solubility in a solution-particle (related to dif- 
fusion and tension, giving rise to solubility spectrum (dilution levels) of a solution-particle in media) has given rise to a particle-in-time to be erroneously attributed to a function governed by probability. These are possibly the Bohm trajectories [11] [12]. This work shows that a particle-in-time is merely a constituent of an elaborate continuous system at the boundary of space and time. A particle-in-time is carried by (unstable) waves. These waves move at different movement rates. The frame wave moves faster than the diffusion and the pilot wave. This work highlights that a particle-in-time is a sophisticated equilibrium maintaining and seeking entity with a potentially evolving neighbourhood accompanied by waves that carry information about its ability to share and acquire properties and also its progression in time.

\section{Acknowledgements}

The author would like to thank the following; C. Nhachi, C. Masimirembwa, and G. Kadzirange, AIBST and The College of Health Sciences, University of Zimbabwe.

\section{References}

[1] Hubert, F.M. and Goenner, H. (2004) On the History of Unified Field Theories. Living Reviews in Relativity, 7, 5-129.

[2] Gross, D. (2005) Einstein and Search for Unification. Current Science, 88, 20352040.

[3] Janssen, M. and Lehner, C. (2014) The Cambridge Companion to Einstein. Cambridge University Press, Cambridge. https://doi.org/10.1017/CCO9781139024525

[4] Bohm, D. (1952) A Suggested Interpretation of the Quantum Theory in Terms of "Hidden" Variables. Physical Review, 85, 66-179. https://doi.org/10.1103/physrev.85.166

[5] Nemaura, T. (2015) Modeling Transportation of Efavirenz: Inference on Possibility of Mixed Modes of Transportation and Kinetic Solubility. Frontiers in Pharmacology, 8, 6-121. https://doi.org/10.3389/fphar.2015.00121

[6] Nemaura, T. (2016) The Advection Wave-in-Secondary Saturation Movement Equation and Its Application to Concentration Tension-Driven Saturation Kinetic Flow. Journal of Applied Mathematics and Physics, 4, 2126-2134. https://doi.org/10.4236/jamp.2016.412210

[7] Nemaura, T. (2016). The Advection Diffusion-in-Secondary Saturation Movement Equation and Its Application to Concentration Gradient-Driven Saturation Kinetic Flow. Journal of Applied Mathematics and Physics, 4, 1998-2010. https://doi.org/10.4236/jamp.2016.411200

[8] Nemaura, T. (2014) Projections of Pharmacokinetic Parameter Estimates from Middose Plasma Concentrations in Individuals on Efavirenz: A Novel Approach. African Journal of Pharmacy and Pharmacology, 8, 929-952.

[9] Hilgevoord, J. (2004) Time in Quantum Mechanics: A Story of Confusion. Studies in History and Philosophy of Modern Physics, 36, 29-60. https://doi.org/10.1016/j.shpsb.2004.10.002

[10] Nemaura, T. (2016) Modelling the Dynamical State of the Projected Primary and Secondary Intra-Solution-Particle Movement System of Efavirenz In Vivo. International Journal of Modern Nonlinear Theory and Application, 5, 235-247. 
https://doi.org/10.4236/ijmnta.2016.54021

[11] Hiley, B.J. (2002) From the Heisenberg Picture to Bohm: A New Perspective on Active Information and Its relation to Shannon Information. Proceedings of Conference Quantum Theory Reconsideration of Foundations, Vaxjo, 6-11 June 2005.

[12] Philippidis, C., Dewdney, C. and Hiley, B.J. (1979) Quantum Interference and the Quantum Potential. Nuovo Cimento B, 52, 15-28. https://doi.org/10.1007/BF02743566

[13] Jagielski, B. (2009) Elements of the Wave-Particle Duality of Light. Master's Thesis, University of Oslo, Oslo.

Submit or recommend next manuscript to SCIRP and we will provide best service for you:

Accepting pre-submission inquiries through Email, Facebook, LinkedIn, Twitter, etc. A wide selection of journals (inclusive of 9 subjects, more than 200 journals)

Providing 24-hour high-quality service

User-friendly online submission system

Fair and swift peer-review system

Efficient typesetting and proofreading procedure

Display of the result of downloads and visits, as well as the number of cited articles

Maximum dissemination of your research work

Submit your manuscript at: http://papersubmission.scirp.org/

Or contact jamp@scirp.org 